\title{
Highly Selective Determination of Dopamine in the Presence of Ascorbic Acid and Serotonin at Glassy Carbon Electrodes Modified with Carbon Nanotubes Dispersed in Polyethylenimine
}

\author{
Marcela C. Rodríguez, María D. Rubianes, and Gustavo A. Rivas* \\ INFIQC, Departamento de Físico Química, Facultad de Ciencias Químicas, Universidad Nacional de Córdoba, \\ Ciudad Universitaria, 5000 Córdoba, Argentina
}

\begin{abstract}
We report the highly selective and sensitive voltammetric dopamine quantification in the presence of ascorbic acid and serotonin by using glassy carbon electrodes modified with a dispersion of multiwall carbon nanotubes (MWCNT) in polyethylenimine, PEI (GCE/MWCNT-PEI). The electrocatalytic activity of the MWCNT deposited on the glassy carbon electrode has allowed an important decrease in the overvoltages for the oxidation of ascorbic acid and dopamine, making possible a clear definition of dopamine, serotonin and ascorbic acid oxidation processes. The sensitivities for dopamine in the presence and absence of $1.0 \mathrm{mM}$ ascorbic acid and serotonin were $(2.18 \pm 0.03) \times 10^{5} \mu \mathrm{AM}^{-1}$ $(r=0.9998)$; and $(2.10 \pm 0.07) \times 10^{5} \mu \mathrm{AM}^{-1}(r=0.9985)$, respectively, demonstrating the excellent performance of the GCE/MWCNT-PEI. The detection limit for dopamine in the mixture was $9.2 \times 10^{-7} \mathrm{M}$. The R. S. D. for the determination of $50 \mu \mathrm{M}$ dopamine using four different electrodes was $3.9 \%$ when modified with the same MWCNT/PEI dispersion, and $4.6 \%$ when using four different dispersions. The modified electrode has been successfully applied for recovery assays of dopamine in human blood serum. Therefore, the new sensor represents an interesting and promising alternative for the electrochemical quantification of neurotransmitters and other analytes of clinical interest.
\end{abstract}

Keywords: Carbon Nanotubes, Glassy Carbon, Catalysis, Polyethylenimine, Carbon Nanotubes Dispersion, Neurotransmitters, Dopamine, Ascorbic Acid, Serotonin, Electrochemical Sensor.

\section{INTRODUCTION}

Dopamine (DA) is an important neurotransmitter present in the central nervous system of mammalian. It plays a very important role in the functioning of central nervous, hormonal, cardiovascular and renal systems. ${ }^{1,2}$ Several alterations in dopaminergic transmission have been related to severe problems like Parkinson disease, schizophrenia and drug dependence. ${ }^{3}$ Therefore, sensitive and selective strategies to determine DA are highly required. In this sense, electroanalytical techniques have been widely used for the quantification of DA due to their known advantages and the easy oxidation of DA at different electrodes, mainly involving carbon materials. ${ }^{3}$

The major drawback of the electrochemical determinations of DA is the interference of easily oxidizable compounds usually present in nervous systems, like ascorbic acid (AA) and serotonin (SER) that are oxidized at

${ }^{*}$ Author to whom correspondence should be addressed. potentials close to those of DA at most of the common electrodes. Several attempts have been made to circumvent this problem. The modification of electrodes with permselective layers has demonstrated to be very successful. ${ }^{4-7}$ The use of electrochemical sensors based on carbon nanotubes (CNT) represents another very advantageous alternative. ${ }^{8,9}$ Zhang et al. ${ }^{10}$ have proposed the use of a glassy carbon electrode (GCE) modified with poly(styrene sulfonic acid)sodium salt/single wall carbon nanotubes for the determination of DA in the presence of excess of AA based on the different electrostatic interaction of these compounds with the polyelectrolyte. Shervedani et al. ${ }^{11}$ have reported the highly selective quantification of DA at micromolar levels in the presence of $1.0 \mathrm{mM}$ AA using a gold electrode modified with cysteamine. Zhu et al. ${ }^{12}$ have proposed the determination of DA in the presence of uric acid (UA) and AA using GCE modified with 1-octyl-3-methylimidazolium hexafluorophosphate containing multi-wall carbon nanotubes (MWCNT). The selective determination of DA in the 
presence of AA using a GCE modified by assembling of poly(diallyldimethylamonium chloride) and negatively charged-shortened-MWCNT has also been demonstrated. ${ }^{13}$ $\mathrm{Hu}$ et al. ${ }^{14}$ have proposed the use of a GCE modified with a dispersion of MWCNTs in water in the presence of dihexadecyl/hydrogen phosphate for the simultaneous determination of DA and serotonin (SER) without interference of AA. They have reported that the fast adsorption of DA and SER on the modified GCE makes possible a very sensitive determination of DA (D.L. $1.1 \times 10^{-8} \mathrm{M}$ ) and SER (D.L. $5 \times 10^{-9} \mathrm{M}$ ) and the quantification of SER in human blood serum after spiking DA and SER. Xie et al. ${ }^{15}$ have proposed the use of gold electrodes modified with overoxidized polypyrrole-multiwalled carbon nanotubes nanocomposite film for the nanomolar detection of DA by Differential Pulse Voltammetry. Chen et al. ${ }^{16}$ have reported the advantages of using a conductive composite film containing MWCNT with poly(methylene blue) synthetized on different electrodes for the simultaneous detection of DA and AA, with detection limit of $67 \mu \mathrm{M}$ DA. The successful use of a platinum electrode modified with single wall carbon nanotubes (SWCNT) and phitic acid to determine DA in the presence of AA and UA, with detection limit of DA of $0.08 \mu \mathrm{M}$, has been proposed. ${ }^{17}$ Glassy carbon electrodes modified with a dispersion of MWCNTs in polyacrylic acid have been also exploited for the highly sensitive and selective quantification of DA $(20 \mathrm{nM})$ and UA $(110 \mathrm{nM})$ in the presence of $0.3 \mathrm{mM}$ AA. ${ }^{18}$ Shahrokhian et al. ${ }^{19}$ have reported the highly sensitive (detection limit $0.08 \mu \mathrm{M}$ ) and selective determination of DA in the presence of AA at carbon paste electrode modified with thionine-Nafion supported on MWCNT.

Recently, we have proposed the highly efficient dispersion of MWCNT in the polycation polyethylenimine (PEI) and the excellent performance of glassy carbon electrodes modified with this dispersion as amperometric detector not only in batch ${ }^{20}$ but also in Flow Injection Analysis and Capillary Electrophoresis. ${ }^{21}$ The irreversible PEI adsorption onto the sidewalls of CNT by wrapping around them is responsible for the highly efficient $n$-doping of SWCNTs due to the electron donating ability of the large numbers of amine groups present in the polymer. ${ }^{22}$ In this work we propose the use of GCE modified with MWCNTs dispersed in PEI as a sensing layer for the highly selective dopamine quantification even in the presence of a large excess of AA and SER.

\section{EXPERIMENTAL DETAILS}

\subsection{Reagents}

Hydrogen peroxide (30\% v/v aqueous solution) was purchased from Baker. Ascorbic acid (AA) was obtained from Fluka. Dopamine (3,4-dihydroxyphenethylamine, DA), serotonin (5-hydroxy tryptamine, SER), and polyethylenimine (PEI, Average MW 750,000, Catalog number
P-3143) were purchased from Sigma. Multi-walled carbon nanotubes powder (MWCNT, 20-50 nm diameter, 5-20 microns length) was obtained from NanoLab (USA). Lyophilized human blood serum (Standatrol) was provided by Wienner. Other chemicals were reagent grade and used without further purification. Ultrapure water $(\rho=18 \mathrm{M} \Omega \mathrm{cm})$ from a Millipore-MilliQ system was used for preparing all the solutions. A $0.050 \mathrm{M}$ phosphate buffer solution $\mathrm{pH} 7.40$ was employed as supporting electrolyte.

\subsection{Apparatus}

The measurements were performed with an EPSILON potentiostat (BAS). The electrodes were inserted into the cell (BAS, Model MF-1084) through holes in its Teflon cover. A platinum wire and $\mathrm{Ag} / \mathrm{AgCl}, 3 \mathrm{M} \mathrm{NaCl}$ (BAS, Model RE-5B) were used as counter and reference electrodes, respectively. All potentials are referred to the latter. A magnetic stirrer provided the convective transport during the amperometric measurements. Scanning Electronic Microscopy (SEM) images were obtained with a Hitachi S3000N Microscope equipped with secondary and backscattered electron detectors. IR experiments were performed with a Nicolet 5-SXC FT-IR spectrometer.

\subsubsection{Preparation of GCE/(MWCNT/PEI)}

Preparation of MWCNT-PEI dispersion: It was obtained by dispersing $1.0 \mathrm{mg}$ of MWCNTs within $1.0 \mathrm{~mL}$ of $1.0 \mathrm{mg} / \mathrm{mL}$ PEI solution (prepared in $50: 50 \mathrm{v} / \mathrm{v}$ ethanol/water) followed by sonication for $15 \mathrm{~min}$.

Preparation of the glassy carbon electrode modified with the MWCNT-PEI dispersion (GCE/(MWCNT-PEI)): The glassy carbon electrode was previously polished with alumina slurries of 1.0, 0.30 and $0.05 \mu \mathrm{m}$ for 2 min each and sonicated in water for $30 \mathrm{~s}$. Then it was modified with the MWCNT-PEI dispersion (GCE/(MWCNT-PEI)) in the following way: an aliquot of $20 \mu \mathrm{L}$ was dropped on top of a polished GCE and then the electrode was left in the air to allow the solvent to evaporate at room temperature (approximately $90 \mathrm{~min}$ in our experimental conditions).

Preparation of the glassy carbon electrode modified with PEI solution (GCE/PEI): It was prepared by dropping $20 \mu \mathrm{L}$ of $1.0 \mathrm{mg} / \mathrm{mL}$ PEI solution (prepared in 50:50 v/v ethanol/water) on polished and clean GCE.

\subsubsection{Preparation of the Samples for IR}

CNTs dispersions for IR were prepared in ethanol/water $50 / 50 \mathrm{~V} / \mathrm{V}$ and sonicated for $15 \mathrm{~min}$. CNT/PEI dispersions were prepared as indicated above.

\subsection{Procedure}

Cyclic voltammetric experiments were performed at $0.100 \mathrm{Vs}^{-1}$. Differential pulse voltammograms (DPV) 
were obtained with a pulse height of $0.040 \mathrm{~V}$ and pulse duration of $200 \mathrm{~ms}$. All the experiments were conducted at room temperature.

\section{RESULTS AND DISCUSSION}

\subsection{Characterization of MWCNT-PEI Dispersion and GCE/(MWCNT-PEI)}

Figure 1 illustrates FT-IR spectra for unmodified MWCNT (A) and MWCNT dispersed in PEI (B). The spectrum for unmodified MWCNT shows a moderate to weak absorption between 1667 and $1640 \mathrm{~cm}^{-1}$, region assigned to $\mathrm{C}=\mathrm{C}$ stretching of phenyl ring vibrations. These peaks are originated from the underlying CNTs structure and can be observed in all CNTs spectra ${ }^{23}$ (The spectrum of was magnified in order to show more clearly the different contributions.). On the contrary, the spectrum corresponding to CNTs dispersed in PEI shows several additional peaks as a consequence of the presence of basic nitrogen-containing groups (e.g., amine) on the CNTs. These peaks are due to the bending vibration of $\mathrm{N}-\mathrm{H}$ (1648, 1567 and $\left.1475 \mathrm{~cm}^{-1}\right)$, stretching vibration of $\mathrm{C}-\mathrm{N}$ $\left(1318,1100\right.$ and $\left.1022 \mathrm{~cm}^{-1}\right)$. Medium to strong absorption was observed between $909-666 \mathrm{~cm}^{-1}$, due to $\mathrm{NH}$ wagging. These functional groups are hydrophilic and make possible the easy dispersion of MWCNTs in aqueous medium. Absorption peaks at $2946 \mathrm{~cm}^{-1}$ and $2850 \mathrm{~cm}^{-1}$ can be assigned to aliphatic $\mathrm{C}-\mathrm{H}$ stretching frequencies of the ethylene group of PEI. Therefore, the results confirm that PEI is effectively wrapped on the MWCNTs walls upon ultrasonication.

Figure 2 shows a SEM image of a GCE coated with the MWCNT/PEI dispersion obtained at 50,000 $\times$. The picture reveals that MWCNTs are efficiently entrapped within the polymeric matrix and distributed in a homogeneous way on the glassy carbon surface.

A quick and efficient way to demonstrate the catalytic activity of MWCNTs dispersed in the polymeric film and

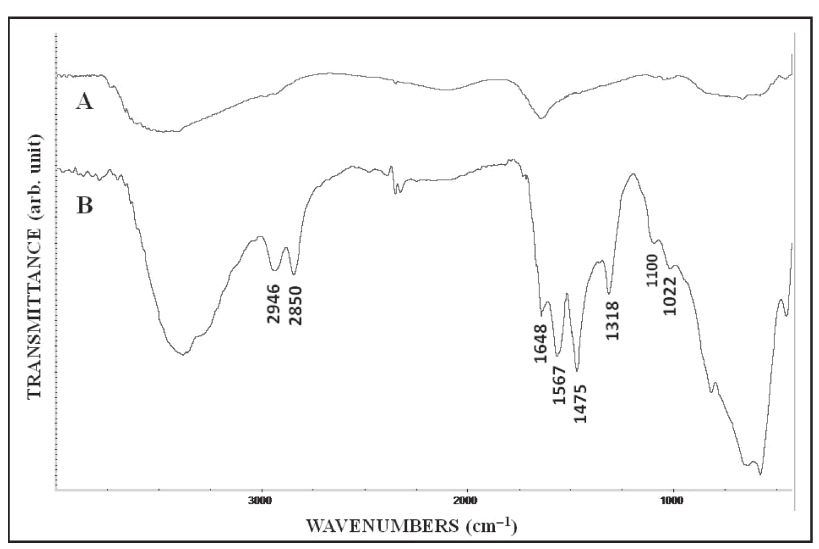

Fig. 1. FT-IR spectra for unmodified MWCNT (A) and MWCNT-PEI (B) (The amount of MWCNT to get the spectrum is higher than that of MWCNT-PEI.).

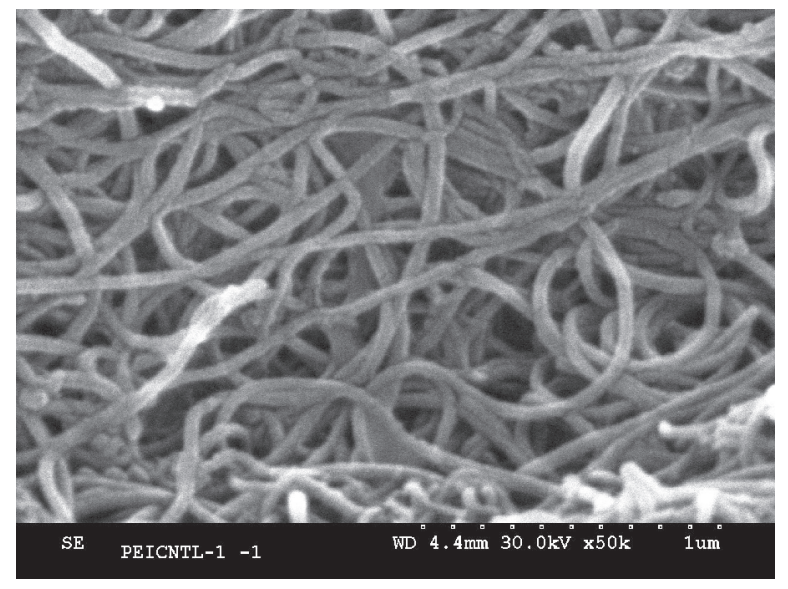

Fig. 2. SEM picture obtained for a GCE modified with the dispersion of CNTs in PEI. Magnification: 50,000×.

deposited on the glassy carbon surface is the evaluation of the voltammetric behavior of hydrogen peroxide at the resulting electrode. Figure 3 shows the voltammetric response of $50 \mathrm{mM}$ hydrogen peroxide at different electrodes: bare glassy carbon electrode (GCE) (a), GCE modified with PEI (GCE/PEI) (b), and GCE modified with the MWCNT-PEI dispersion (GCE/MWCNT-PEI) (c). In a way similar to that observed at GCE (a), elevated overvoltages are necessary to oxidize and reduce hydrogen peroxide at GCE/PEI (b). On the contrary, when MWCNTs are present at the electrode (GCE/MWCNT-PEI), an important decrease in the oxidation and reduction overvoltages is obtained as a consequence of the electrocatalytic activity of MWCNT, in agreement with previous results. ${ }^{20,21}$ As it was previously reported, ${ }^{20,21}$ the MWCNT-PEI dispersion is highly stable. Electrodes prepared with the same dispersion even 14 days after the first day, gave the same response as the one obtained the first day of the dispersion. ${ }^{20}$ GCE/MWCNT-PEI also demonstrated to be highly stable in flow experiments performed either through

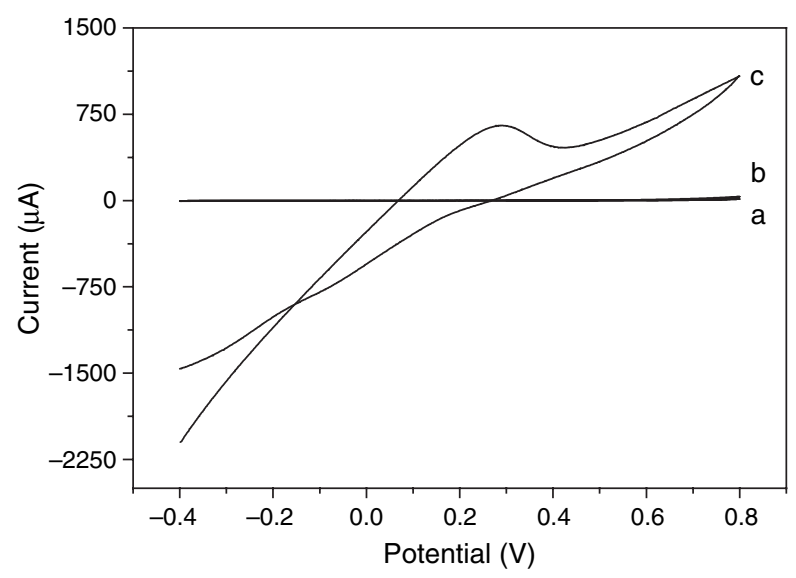

Fig. 3. Cyclic voltammogram for $50 \mathrm{mM}$ hydrogen peroxide at different electrodes: GCE (a), GCE-PEI (b), and GCE/MWCNT-PEI (c). Scan rate: $0.100 \mathrm{Vs}^{-1}$. Supporting electrolyte: $0.050 \mathrm{M}$ phosphate buffer solution $\mathrm{pH} 7.40$. 
continuous flow (for $45 \mathrm{~min}$ ) or successive additions (30) of amitrol, phenol, dopamine and dopac. ${ }^{21}$

\subsection{Electrochemical Oxidation of Dopamine, Ascorbic Acid and Serotonin}

Figure 4 shows cyclic voltammograms for $1.0 \mathrm{mM}$ DA (A), AA (B) and SER (C) at GCE (a), GCE/PEI (dashed line, b)
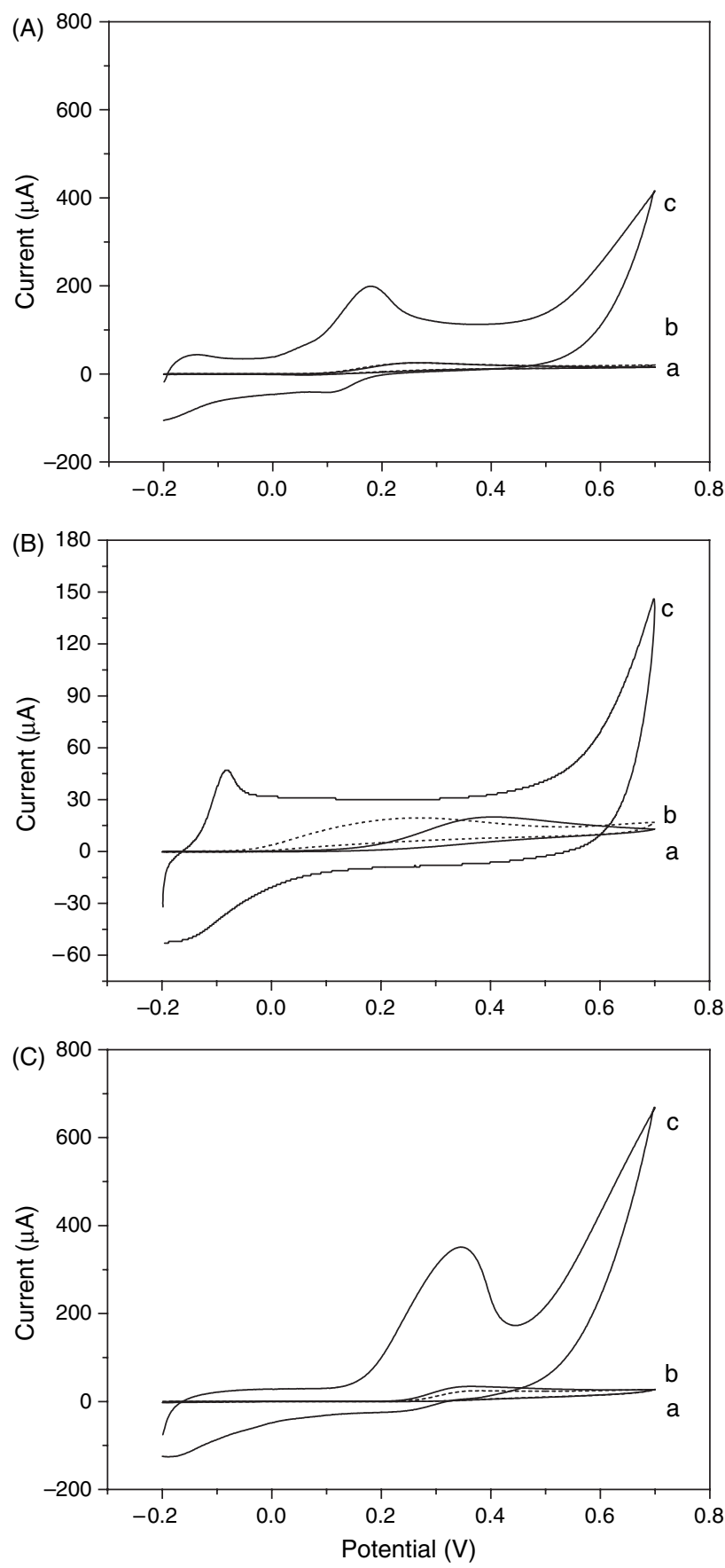

Fig. 4. Cyclic voltammograms for $1.0 \mathrm{mM}$ dopamine (A), ascorbic acid (B) and serotonin (C) at different electrodes: GCE (a), GCE-PEI (b), and GCE/MWCNT-PEI (c). Scan rate: $0.100 \mathrm{Vs}^{-1}$. Supporting electrolyte: $0.050 \mathrm{M}$ phosphate buffer solution $\mathrm{pH} 7.40$. and GCE/MWCNT-PEI (c). A decrease of $90 \mathrm{mV}$ in the peak potential separation and an increase of around 8-fold in the oxidation current is observed at GCE/MWCNT-PEI. A better definition of the dopaminequinone reduction peak and associated redox processes is also evident.

Figure 4(B) depicts cyclic voltammograms for AA at the three different electrodes. A shifting of $140 \mathrm{mV}$ in the negative direction is observed for the oxidation peak potential at GCE-PEI (dashed line), due to the favored electrostatic interaction of ascorbate with the polycation. When MWCNT are present, a shifting of $490 \mathrm{mV}$ in the negative direction for AA oxidation peak potential and an increase of around 50\% in the current are observed (compared to GCE).

Cyclic voltammograms for SER obtained at the different electrodes are shown in Figure 4(C). At bare GCE the voltammogram shows an oxidation peak at $0.366 \mathrm{~V}$, with no reduction peak. In the presence of PEI, the oxidation peak potential remains almost constant while the current decreases (34.3 vs. $24.5 \mu \mathrm{A}$ at GCE and GCE/PEI, respectively) due to some electrostatic repulsion with the positively charged PEI. In the presence of MWCNT the oxidation peak potential decreases $20 \mathrm{mV}$ and the current largely increases (34.3 vs. $351.8 \mu \mathrm{A}$ at GCE and GCE/MWCNT-PEI, respectively).

In summary, the modification of GCE with MWCNTPEI largely improves the electro-oxidation of AA, DA and SER, even when the capacitive currents are higher than those at GCE and GCE/PEI. This behavior can be attributed to the electrocatalytic activity of CNTs efficiently dispersed in PEI and successfully deposited on the surface of GCE and to the significant increase in the surface area of the resulting electrodes.

\subsection{Simultaneous Determination of DA and AA}

Figure 5(A) shows DPVs for a mixture of $1.0 \mathrm{mM}$ DA and $1.0 \mathrm{mM} \mathrm{AA}$ at bare GCE (A), and GCE/MWCNT-PEI (B). As expected, a broad peak is obtained at the bare electrode, indicating that is not possible to detect a mixture of these compounds. Figure 5(B) displays the voltammetric response for the same mixture $(1.0 \mathrm{mM}$ DA and $1.0 \mathrm{mM}$ $\mathrm{AA})$ at GCE/MWCNT-PEI. Two very well-defined DPV peaks at $-0.108 \mathrm{~V}$ and $0.128 \mathrm{~V}$ are observed for the oxidation of AA and DA, respectively. Therefore, the catalytic activity of MWCNT allows a clear definition of the two oxidation processes as well as a significant enhancement in the associated currents, ensuring, in that way, the individual determination of the two compounds. At GCE/PEI the signals are smaller and the resolution is very poor (not shown).

Considering the interesting results shown in Figure 5, we evaluate the feasibility to quantify DA in the presence of a large excess of AA using GCE/MWCNTPEI. Figure 6(A) shows DPV recordings obtained at 

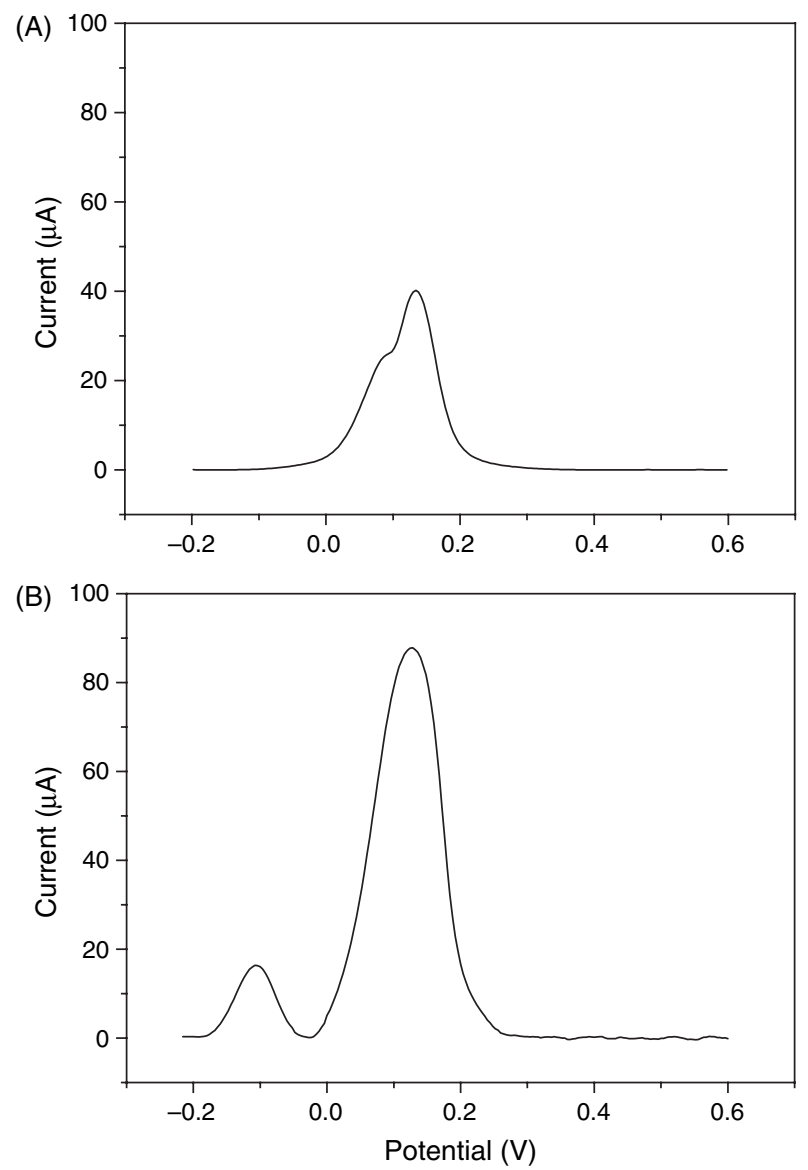

Fig. 5. Differential pulse voltammograms for mixtures containing $1.0 \mathrm{mM}$ dopamine and ascorbic acid at GCE (A) and GCE/MWCNT-PEI (B). Scan rate: $0.100 \mathrm{Vs}^{-1}$. Pulse height: $0.040 \mathrm{~V}$. Pulse duration: $200 \mathrm{~ms}$. Supporting electrolyte: $0.050 \mathrm{M}$ phosphate buffer solution $\mathrm{pH} 7.40$.

GCE/MWCNT-PEI for mixtures of DA and AA containing increasing concentrations of DA from 10 to $100 \mu \mathrm{M}$ and $1.0 \times 10^{-3} \mathrm{M}$ AA. In agreement with the results shown in Figure 5(B), two clearly separated peaks are obtained at $-0.120 \mathrm{~V}$ and $0.090 \mathrm{~V}$ for $\mathrm{AA}$ and $\mathrm{DA}$, respectively. A slight shifting in the positive direction is observed for DA oxidation peak potential as the concentration increases, while the peak potentials and currents for the oxidation of AA remain constant. Figure 6(B) compares calibration plots for DA obtained in the absence (empty circles) and in the presence (full circles) of $\mathrm{AA}$, as well as the corresponding response for AA in the presence of different concentrations of DA (triangles). It is important to remark that every experiment was obtained with a new electrode and that each point represents the average of the currents obtained with three different electrodes. The signal for AA remains constant even for the solution containing $100 \mu \mathrm{M}$ DA. The sensitivities for DA obtained in the presence and absence of AA are $(2.16 \pm 0.05) \times 10^{5} \mu \mathrm{AM}^{-1}(r=0.998)$; and $(2.25 \pm$ $0.03) \times 10^{5} \mu \mathrm{AM}^{-1}(r=0.998)$, respectively. This small difference in sensitivity for DA at GCE/MWCNT-PEI in
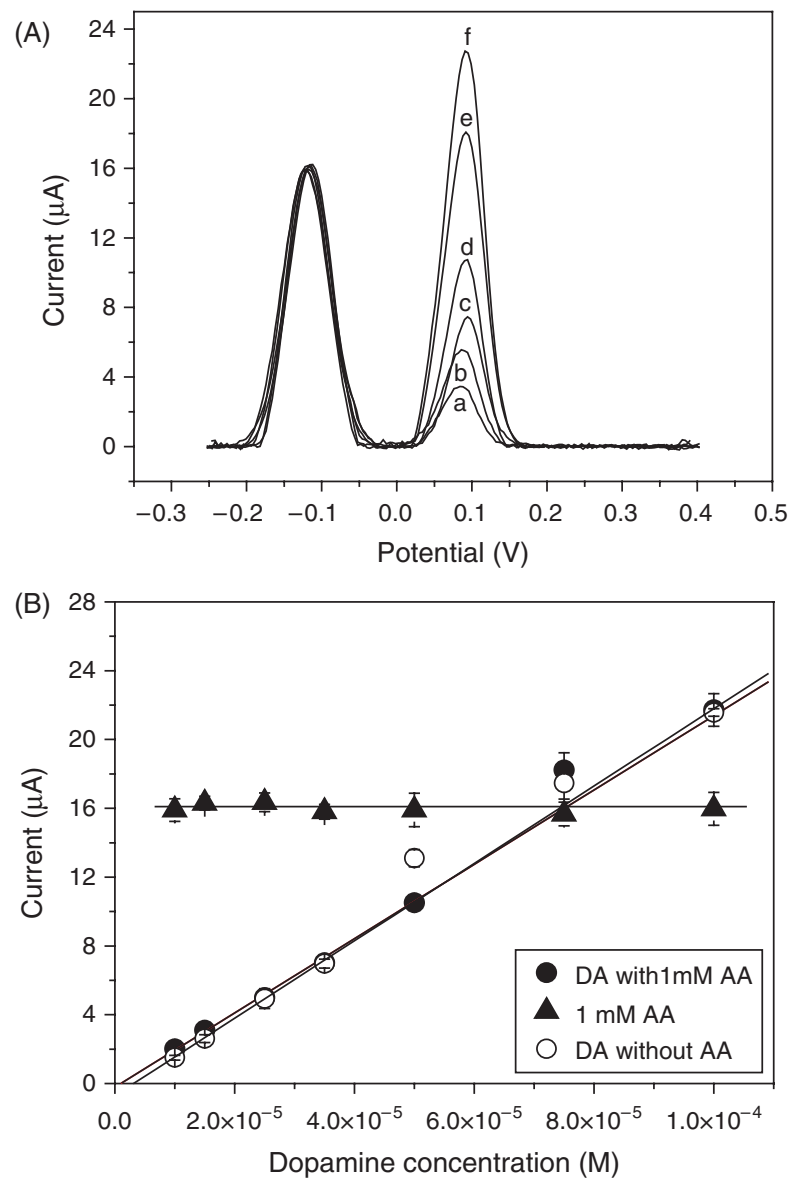

Fig. 6. (A) Differential pulse voltammograms for mixtures containing $1.0 \mathrm{mM}$ ascorbic acid and different concentrations of dopamine: 10 (a), 25 (b), 35 (c), 50 (d), 75 (e) and 100 (f) $\mu \mathrm{M}$. (B) Current versus dopamine concentration plot for dopamine in the presence (full circle) and absence (empty circle) of ascorbic acid; as well as for ascorbic acid (triangle) in the presence of the different concentrations of dopamine. Other conditions as in Figure 5.

the absence and presence of AA (4.2\%), clearly demonstrate the feasibility to determine DA and AA in mixtures of both compounds using the GCE/MWCNT-PEI without any interference, even for 100-fold excess of AA compared to DA and using an AA concentration as high as $1.0 \mathrm{mM}$.

In a similar way, determinations of AA in the presence of $1.0 \mathrm{mM}$ Do are also possible. Figure 7(A) shows DPVs for increasing concentrations of AA from 0.50 to $5.0 \mathrm{mM}$ in the presence of $1.0 \mathrm{mM}$ DA. Two clearly resolved voltammetric peaks can be observed in all cases. Figure 7(B) displays calibration plots for AA at GCE/MWCNT-PEI in the absence (empty circles) and in the presence (full circles) of DA, as well as the response for DA for the solutions containing different concentrations of AA in the mixture (triangles). The peak current for DA remains constant even for the highest concentration of AA in the solution. The sensitivities for AA obtained in the presence and absence of DA are $(1.6 \pm 0.3) \times 10^{4}(r=0.9991)$, and $(1.5 \pm 0.5) \times$ $10^{4} \mu \mathrm{AM}^{-1}(r=0.998)$, respectively, showing a difference just of $6.2 \%$. The signal for DA remains constant even for 

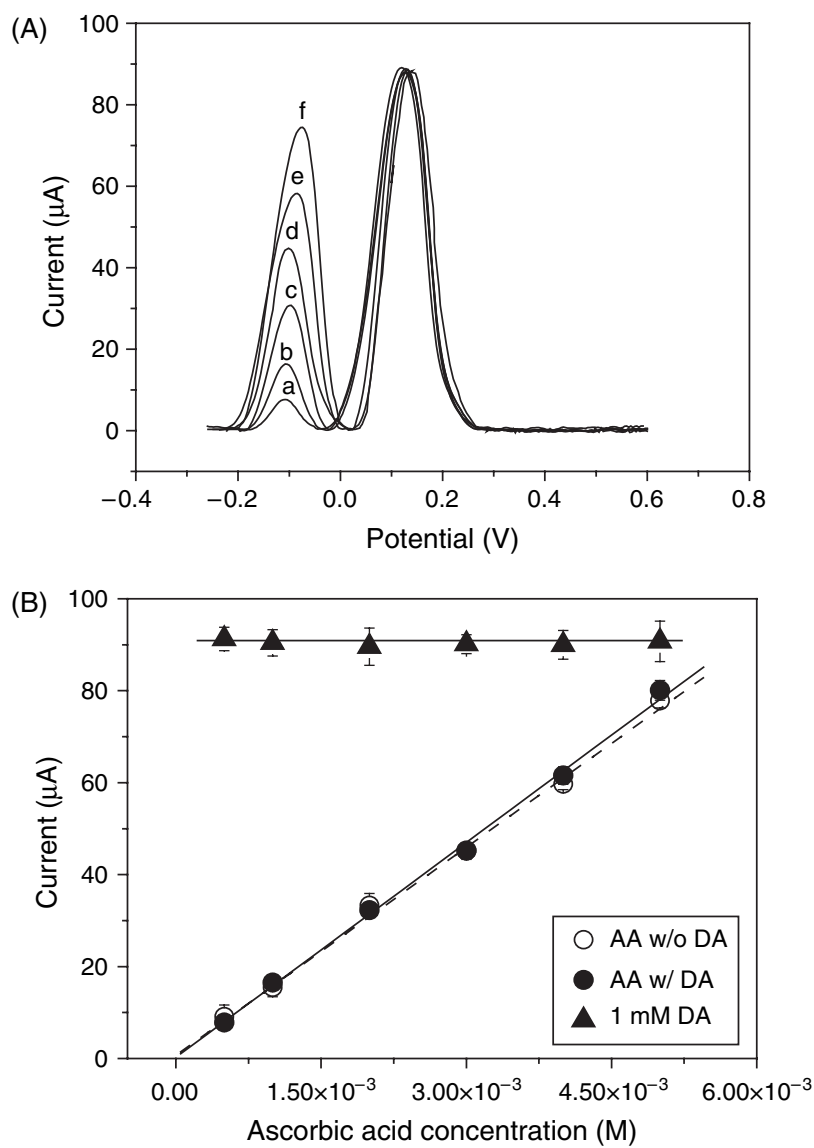

Fig. 7. (A) Differential pulse voltammograms for mixtures containing $1.0 \mathrm{mM}$ dopamine and different concentrations of ascorbic acid: 0.50 (a), 1.00 (b), 2.00 (c), 3.00 (d), 4.00 (e) and 5.00 (f) mM. (B) Current versus ascorbic acid concentration plot for ascorbic acid in the presence (full circle) and absence (empty circle) of dopamine; as well as for dopamine (triangle) in the presence of the different concentrations of ascorbic acid. Other conditions as in Figure 5.

$5.0 \mathrm{mM}$ AA, clearly indicating the feasibility to quantify AA in the presence of DA.

\subsection{Determination of DA in the Presence of AA and SER}

Figure 8(A) shows DPVs for mixtures containing $1.0 \mathrm{mM}$ AA, $1.0 \mathrm{mM}$ SER and increasing concentrations of DA from 10 to $100 \mu \mathrm{M}$ at GCE/MWCNT-PEI. At variance with the voltammograms obtained at GCE, where just two peaks were obtained (not shown), at GCE/MWCNT-PEI the signal is resolved into three well-defined DPV peaks at $-0.120 \mathrm{~V}, 0.090 \mathrm{~V}$ and $0.270 \mathrm{~V}$ for the oxidation of AA, DA, and SER, respectively. Once more, the presence of MWCNT ensures a highly sensitive response and an adequate shifting in the peak potential for the oxidation of $\mathrm{AA}$, and makes possible the individual determination of the three compounds.

The concentration of DA in the mixtures can be determined even in the presence of $1.0 \mathrm{mM} \mathrm{AA}$ and $1.0 \mathrm{mM}$ SER. Figure 8(B) depicts calibration plots for DA at
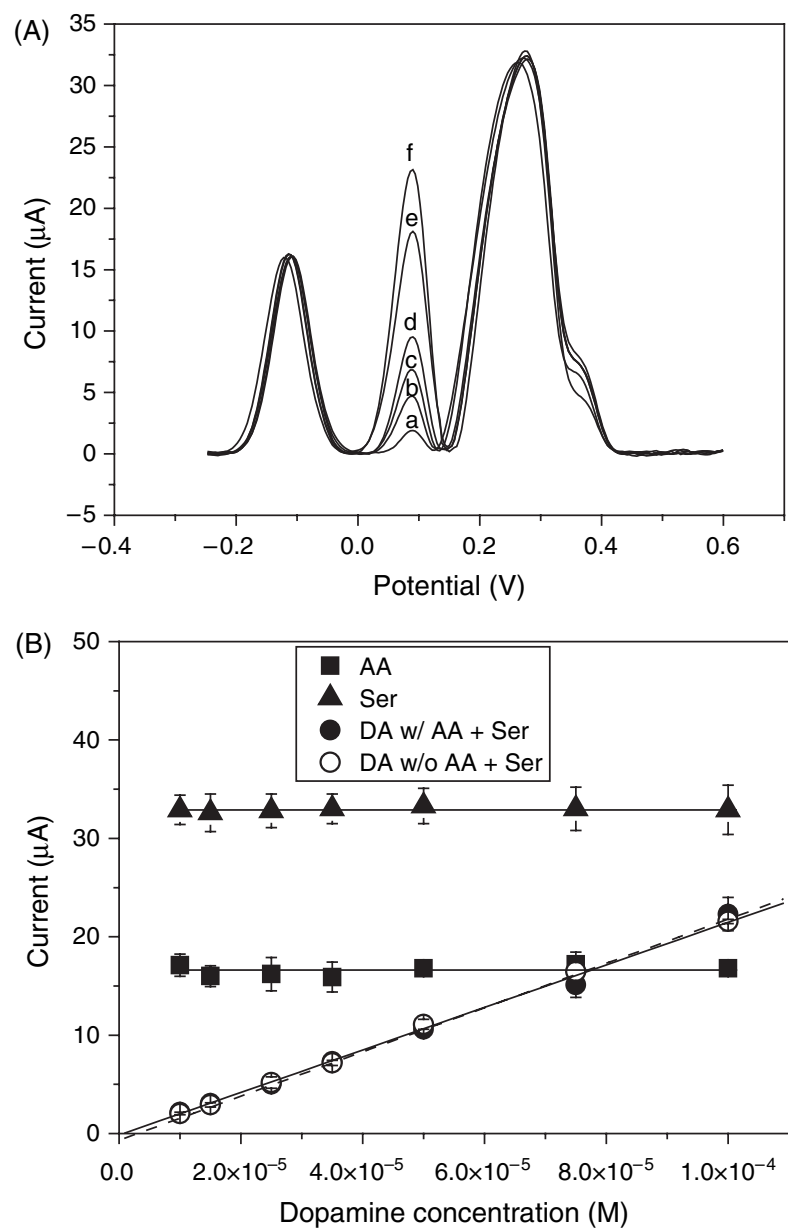

Fig. 8. (A) Differential pulse voltammograms for mixtures containing $1.0 \mathrm{mM}$ ascorbic acid, $1.0 \mathrm{mM}$ serotonin and different concentrations of dopamine: 10 (a), 25 (b), 35 (c), 50 (d), 75 (e) and 100 (f) $\mu$ M. (B) Current versus dopamine concentration plot for dopamine in the presence (full circle) and absence (empty circle) of ascorbic acid and serotonin; as well as for ascorbic acid (square) and serotonin (triangle) in the presence of the different concentrations of dopamine. Other conditions as in Figure 5.

GCE/MWCNT-PEI in the presence (full circles) and in the absence (empty circles) of SER and AA. The sensitivities for DA in the presence and absence of AA and SER are $(2.18 \pm 0.03) \times 10^{5} \mu \mathrm{AM}^{-1}(r=0.9998)$; and $(2.10 \pm$ $0.07) \times 10^{5} \mu \mathrm{AM}^{-1}(r=0.9985)$, respectively, representing a difference in sensitivities in the absence and presence of AA and SER just of 3.7\%. As it is also shown in Figure 8, the signals for AA (squares) and SER (triangles) remain constant in the presence of the different concentrations of DA, indicating the feasibility of the method to determine DA in such a complex mixture.

The detection limit for DA in the mixture, obtained as 3.3 times the ratio between standard deviation of the blank and sensitivity, was $9.2 \times 10^{-7} \mathrm{M}$. As it was shown in calibration plots shown in Figures 6, 7 and 8, the analytical methodology was highly reproducible. The R.S.D. for the determination of $50 \mu \mathrm{M}$ DA using four different electrodes modified with the same dispersion was $3.9 \%$. Analogous 


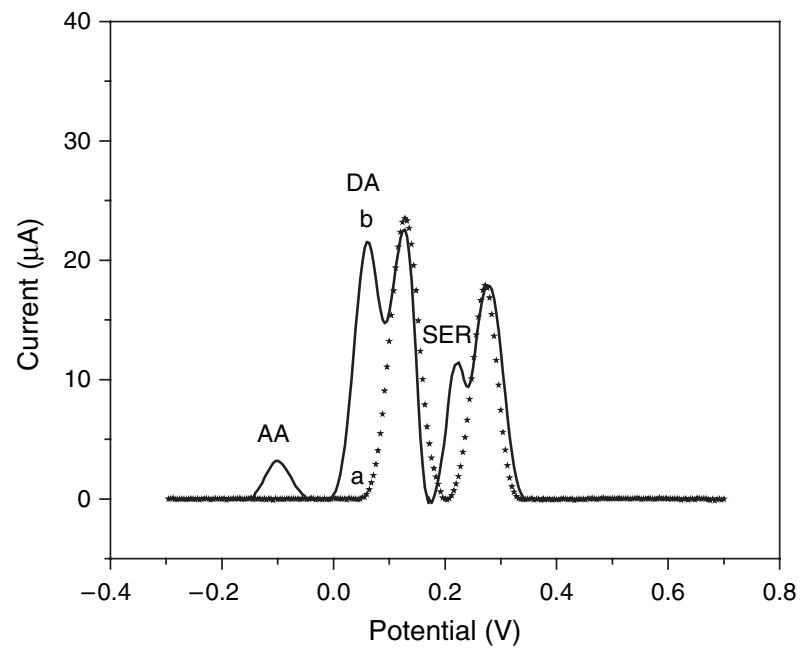

Fig. 9. Differential pulse voltammograms for undiluted blood serum sample before (a) and after (b) the addition of $100 \mu \mathrm{M} \mathrm{AA}+100 \mu \mathrm{M}$ $\mathrm{DA}+100 \mu \mathrm{M}$ SER. Other conditions as in Figure 5.

experiments using four electrodes prepared with four different dispersions gave a R.S.D. of $4.6 \%$.

The usefulness of the proposed methodology for determinations in human blood serum was also evaluated. Figure 9 shows DPVs for undiluted human blood serum before (a) and after (b) the addition of $100 \mu \mathrm{M} \mathrm{AA}+$ $100 \mu \mathrm{M}$ DA $+100 \mu \mathrm{M}$ SER. Experiments performed by separate additions of AA, DA and SER to the blood serum sample demonstrated that the oxidation of these compounds occurred at $-0.09 \mathrm{~V}, 0.06 \mathrm{~V}$ and $0.22 \mathrm{~V}$, respectively (not shown). Therefore, the contributions of AA, DA and SER can be clearly distinguished, making possible the determination of these compounds even in a matrix as complex as blood serum sample. Separate additions of uric acid to the buffer solution or serum sample demonstrated that the peak at $0.13 \mathrm{~V}$ can be attributed to the oxidation of uric acid present in the serum sample (not shown). Additions of $50 \mu \mathrm{M}$ and $100 \mu \mathrm{M}$ DA to undiluted serum samples gave recoveries of $104 \%$ and $99 \%$, respectively.

Adsorptive stripping experiments of DA at CNTmodified-GCE $(5 \mathrm{~min}$ accumulation at $-0.250 \mathrm{~V})$ with medium exchange demonstrated that DA can be preconcentrated at the CNTs-modified GCE, although no improvement in sensitivity was obtained under these conditions compared to the direct DA determination (not shown).

\section{CONCLUSIONS}

In summary, the MWCNT/PEI film-coated GCE exhibits remarkable electrocatalytic effects on the oxidation of DA,
AA and SER, improving their oxidation peak currents and lowering their oxidation overpotentials. At variance with GCE, the GCE modified with MWCNT-PEI film permits a very favorable voltammetric resolution of DA, AA and SER oxidation processes. The GCE/MWCNT-PEI demonstrated to be highly efficient in detecting DA in the presence of large excess of AA and SER. These characteristics make GCE/MWCNT-PEI a novel analytical tool for the selective and sensitive quantification of DA in the presence of AA and SER, and open the doors to new challenges in the electroanalytical determination of other neurotransmitters and further practical applications.

Acknowledgments: The authors thank Fundación Antorchas, CONICET, SECyT-UNC, ANPCyT y Becas Banco Santander-Río for the financial support.

\section{References and Notes}

1. H. C. Fibiger, Mesolimbic Semmin. Neurosci. 5, 321 (1993).

2. P. E. M. Phillips, G. D. Stuber, M. L. A. V. Hein, R. M. Wightman, and R. M. Carelli, Nature 422, 614 (2003).

3. B. J. Venton and R. M. Wightman, Anal. Chem. 414A (2003).

4. M. D. Rubianes and G. A. Rivas, Anal. Chim. Acta 440, 99 (2001).

5. H. Wang, L.-J. Wang, Z.-F. Shi, Y. Guo, X.-P. Cao, and H.-L. Zhang, Electrochem. Comm. 8, 1779 (2006).

6. H. Zhao, Y. Zhang, and Z. Yuan, Analyst 126, 358 (2001).

7. J. M. Mo and B. Ogorevc, Anal. Chem. 73, 1196 (2001).

8. G. A. Rivas, M. D. Rubianes, M. L. Pedano, N. F. Ferreyra, G. L. Luque, S. A. Miscoria, and M. C. Rodríguez, Electroanal. 19, 823 (2007).

9. G. A. Rivas, M. D. Rubianes, M. C. Rodríguez, N. F. Ferreyra, M. L. Pedano, G. L. Luque, S. A. Miscoria, and C. Parrado, Talanta 74, 291 (2007).

10. Y. Zhang, Cai, and S. Su, Anal. Biochem. 350, 285 (2006).

11. R. K. Shervedani, M. Bagherzadeh, and S. A. Mozaffari, Sens. Act. $B$ 115, 614 (2006).

12. Y. Zhao, Y. Gao, D. Zhan, H. Liu, Q. Zhao, Y. Kou, Y. Shao, M. Li, Q. Zhuang, and Z. Zhu, Talanta 66, 51 (2005).

13. M. Zhanf, K. Gong, H. Zhang, and L. Mao, Biosens. Bioelectrons. 20, 1270 (2005).

14. K. Wu, J. Fei, and S. Hu, Anal. Biochem. 318, 100 (2003).

15. X. Tu, Q. Xie, S. Jiang, and S. Yao, Biosens. Bioelectrons. 22, 2819 (2007).

16. U. Yogeswaran and S.-M. Chen, Sens. Act. B 130, 739 (2008).

17. S. Jo, H. Jeong, S. R.-Bae, and S. Jeon, Microchemical Journal 88, 1 (2008).

18. A. Liu, I. Honma, and H. Zhou, Biosens. Bioelectrons. 23, 74 (2007).

19. S. Shahrokhian and H. R. Zare-Mehrjardi, Electrochimica Acta 52, 6310 (2007).

20. M. D. Rubianes and G. A. Rivas, Electrochem. Comm. 9, 480 (2007).

21. A. S. Arribas, E. Bermejo, M. Chicharro, A. Zapardiel, G. Luque, N. Ferreyra, and G. A. Rivas, Anal. Chim. Acta 596, 183 (2007).

22. M. Shim, A. Javey, N. W. S. Kam, and H. H. Dai, J. Am. Chem. Soc. 123, 11512 (2001).

23. M. Aizawa and M. S. P. Shaffer, Chem. Phys. Lett. 368, 121 (2003). 